\title{
Generalized Schmidt Decomposition and Classification of Three-Quantum-Bit States
}

\author{
A. Acín, ${ }^{1}$ A. Andrianov, ${ }^{1,3}$ L. Costa, ${ }^{2}$ E. Jané, ${ }^{1, *}$ J. I. Latorre, ${ }^{1}$ and R. Tarrach ${ }^{1}$ \\ ${ }^{1}$ Departament d'Estructura i Constituents de la Matèria, Universitat de Barcelona, Diagonal 647, E-08028 Barcelona, Spain \\ ${ }^{2}$ Departament d'Àlgebra i Geometria, Universitat de Barcelona, Gran Via Corts Catalanes 585, E-08007 Barcelona, Spain \\ ${ }^{3}$ Department of Theoretical Physics, St. Petersburg State University, 198904, St. Petersburg, Russia
}

(Received 24 March 2000)

\begin{abstract}
We prove for any pure three-quantum-bit state the existence of local bases which allow one to build a set of five orthogonal product states in terms of which the state can be written in a unique form. This leads to a canonical form which generalizes the two-quantum-bit Schmidt decomposition. It is uniquely characterized by the five entanglement parameters. It leads to a complete classification of the threequantum-bit states. It shows that the right outcome of an adequate local measurement always erases all entanglement between the other two parties.
\end{abstract}

PACS numbers: 03.67.-a, 03.65.Bz

The Schmidt decomposition [1,2] allows one to write any pure state of a bipartite system as a linear combination of biorthogonal product states or, equivalently, of a nonsuperfluous set of product states built from local bases. For two quantum bits (qubits) it reads

$$
|\Psi\rangle=\cos \theta|00\rangle+\sin \theta|11\rangle, \quad 0 \leq \theta \leq \pi / 4 .
$$

Here $|i i\rangle \equiv|i\rangle_{A} \otimes|i\rangle_{B}$, both local bases $\{|i\rangle\}_{A, B}$ depend on the state $|\Psi\rangle$, the relative phase has been absorbed into any of the local bases, and the state $|00\rangle$ has been defined by carrying the larger (or equal) coefficient. A larger value of $\theta$ means more entanglement. The only entanglement parameter, $\theta$, plus the hidden relative phase, plus the two parameters which define each of the two local bases are the six parameters of any two-qubit pure state, once normalization and global phase have been disposed of.

Very many results in quantum information theory have been obtained with the help of the Schmidt decomposition: its simplicity reflects the simplicity of bipartite systems as compared to $N$-partite systems. Much of its usefulness comes from it not being superfluous: to carry one entanglement parameter one needs only two orthogonal product states built from local bases states, no more, no less.

The aim of this work is to generalize the Schmidt decomposition of (1) to three qubits. It is well known [2] that its straightforward generalization, that is, in terms of triorthogonal product states, is not possible (see also [3]). Nevertheless, having a minimal canonical form in which to cast any pure state, by performing local unitary transformations, will provide a new tool for quantifying entanglement for three qubits, a notoriously difficult problem. It will lead to a complete classification of exceptional states which, as we will see, is much more complex than in the two-qubit case. The generalization to $N$ quantum dits ( $d$-state systems) is not completely straightforward and will be given elsewhere.

Linden and Popescu [4] and Schlienz [5] showed that for any pure three-qubit state the number of entanglement parameters is five and, using repeatedly the two-qubit
Schmidt decomposition, proved the existence for any pure state of a reference form in terms of six orthogonal product states built from local bases. The five entanglement parameters are one phase (all others can be absorbed) and four moduli of the six coefficients, so that a further constraint beyond the normalization exists. In other words, exactly as (1) shows that local unitary transformations allow one to make two of the four components vanish (corresponding to $|01\rangle$ and $|10\rangle$ ) for a two-qubit pure state, Linden and Popescu and Schlienz proved that, also for a three-qubit system two of the, now eight, components can be made zero. However, the set of six states is superfluous in the sense that its coefficients require a constraint to lead to a unique representative of any pure state. It is not clear whether this is the best one can do, i.e., whether the set is minimal. We will now prove that indeed, combining adequately the local changes of bases corresponding to $\mathrm{U}(1) \times \mathrm{SU}(2) \times \mathrm{SU}(2) \times \mathrm{SU}(2)$ transformations, one can always do with five terms, which precisely can carry only five entanglement parameters, leading thus to a nonsuperfluous unique representation.

Notice that a straightforward counting of parameters shows that a nonsuperfluous set will have five states, i.e., three vanishing coefficients. There exist three inequivalent sets of five local bases product states

$$
\begin{aligned}
& \{|000\rangle,|001\rangle,|010\rangle,|100\rangle,|111\rangle\}, \\
& \{|000\rangle,|001\rangle,|110\rangle,|100\rangle,|111\rangle\}, \\
& \{|000\rangle,|100\rangle,|110\rangle,|101\rangle,|111\rangle\} .
\end{aligned}
$$

Whereas the first set is symmetric under permutation of parties, the other two are not.

The nonequivalence of the three sets follows from the different degrees of orthogonality between the five states within each set. One can also readily check that all three sets can carry exactly five entanglement parameters, four moduli, and one phase, and are thus nonsuperfluous. This is of course no proof that any state can always be written as a linear combination of the five states of one and the same 
set. We will now prove that it can always be done for the last two sets, or their versions obtained by permuting parties.

As an introduction let us first present a one-line proof of the Schmidt decomposition of a two-qubit state, Eq. (1). Writing any state in a basis of product states built from any two local bases,

$$
|\Psi\rangle=\sum_{i, j} t_{i j}|i j\rangle,
$$

calling $T$ the matrix of elements $t_{i j}$, and recalling that for any $T$ there always exist two unitary matrices which diagonalize it,

$$
U_{1} T U_{2}=D
$$

the Schmidt decomposition follows at once. Note that $U_{1}$ and $U_{2}$ correspond to the local basis changes necessary for casting the original state into its Schmidt form.

For a three-qubit state the proof goes as follows: from

$$
|\Psi\rangle=\sum_{i, j, k} t_{i j k}|i j k\rangle
$$

one introduces the matrices $T_{0}$ and $T_{1}$ with elements

$$
\left(T_{i}\right)_{j k} \equiv t_{i j k} \text {. }
$$

Consider now the unitary transformation on the first qubit,

$$
T_{i}^{\prime}=\sum_{j} u_{i j} T_{j}
$$

such that

$$
\operatorname{det} T_{0}^{\prime}=0 .
$$

Notice that (8) has always two solutions. The matrix obtained from $T_{0}^{\prime}$ after diagonalization following (4), which corresponds to unitary transformations on the last two qubits, has at least three zeros,

$$
\left(D_{0}^{\prime}\right)_{01}=\left(D_{0}^{\prime}\right)_{10}=\left(D_{0}^{\prime}\right)_{11}=0 .
$$

This finishes the proof that any pure state of three qubits can always be written as a linear superposition of the five states of the last set of (2).

The generalization to three qubits of the Schmidt decomposition, i.e., one more zero for one more qubit, thus reads

$$
\lambda_{i} \geq 0,0 \leq \varphi \leq \pi, \mu_{i} \equiv \lambda_{i}^{2}, \sum_{i} \mu_{i}=1,
$$

where we have chosen the second coefficient to carry the only relevant phase, whose range, to be proven later, is also given. Notice that we have singled out party $A$ in obtaining (10), but we could have chosen any of the three parties.

An immediate and important consequence of this decomposition is that there always exists for any state $|\Psi\rangle$ and any (genderless) party $X$ a state $|0\rangle_{X}$ such that ${ }_{X}\langle 0 \mid \Psi\rangle$ is a product state of the other two parties (unless party $X$ is not entangled with the other two parties). That is, party $X$, knowing $|\Psi\rangle$, can perform a local measurement which, for one outcome, allows it to be sure that the other two parties share no entanglement whatsoever. Note that when (8) displays two different solutions, two such states exist. This property suggests some applications to quantum information processing. It also leads to an efficient algorithm for computing the $\lambda$ 's and $\varphi$.

There is one small hitch left: as (8) has generically two different solutions, any state can be written in the form of (10) with two different sets of coefficients. Let us dispose generically of this redundancy. Recall that after diagonalization of $T_{0}^{\prime}$ we are left with the matrices

$$
M_{0} \equiv D_{0}^{\prime}=\left(\begin{array}{cc}
\lambda_{0} & 0 \\
0 & 0
\end{array}\right), \quad M_{1}=\left(\begin{array}{cc}
e^{i \varphi} \lambda_{1} & \lambda_{2} \\
\lambda_{3} & \lambda_{4}
\end{array}\right),
$$

for one solution of Eq. (8) and

$$
\tilde{M}_{0}=\left(\begin{array}{cc}
\tilde{\lambda}_{0} & 0 \\
0 & 0
\end{array}\right), \quad \tilde{M}_{1}=\left(\begin{array}{cc}
e^{i \tilde{\varphi}} \tilde{\lambda}_{1} & \tilde{\lambda}_{2} \\
\tilde{\lambda}_{3} & \tilde{\lambda}_{4}
\end{array}\right),
$$

for the other solution. Of course, both solutions can be related by a $\mathrm{U}(1) \times \mathrm{SU}(2) \times \mathrm{SU}(2) \times \mathrm{SU}(2)$ transformation:

$$
\begin{gathered}
\tilde{M}_{0}=e^{i \omega} U_{1}\left(u_{00} M_{0}+u_{01} M_{1}\right) U_{2}, \\
\tilde{M}_{1}=e^{i \omega} U_{1}\left(-u_{01}^{*} M_{0}+u_{00}^{*} M_{1}\right) U_{2},
\end{gathered}
$$

and the inverse

$$
\begin{aligned}
& M_{0}=e^{-i \omega} U_{1}^{\dagger}\left(u_{00}^{*} \tilde{M}_{0}-u_{01} \tilde{M}_{1}\right) U_{2}^{\dagger}, \\
& M_{1}=e^{-i \omega} U_{1}^{\dagger}\left(u_{01}^{*} \tilde{M}_{0}+u_{00} \tilde{M}_{1}\right) U_{2}^{\dagger} .
\end{aligned}
$$

The condition $\operatorname{det} M_{0}=\operatorname{det} \tilde{M}_{0}=0$ leads to

$$
u_{00}=-\frac{\operatorname{det} M_{1}}{\lambda_{0} \lambda_{4}} u_{01} \quad u_{00}^{*}=\frac{\operatorname{det} \tilde{M}_{1}}{\tilde{\lambda}_{0} \tilde{\lambda}_{4}} u_{01} .
$$

It is tedious, but straightforward, to solve the previous equations. Here we need only the following results:

$$
\lambda_{0} \lambda_{4}=\tilde{\lambda}_{0} \tilde{\lambda}_{4}, \quad u_{01}^{*}=-u_{01},
$$

which, from Eq. (15), imply

$$
\operatorname{det} M_{1}=\left(\operatorname{det} \tilde{M}_{1}\right)^{*} \text {. }
$$

From here it follows that

$$
\begin{aligned}
& 0<\varphi<\pi \Leftrightarrow \pi<\tilde{\varphi}<2 \pi, \\
& 0<\tilde{\varphi}<\pi \Leftrightarrow \pi<\varphi<2 \pi .
\end{aligned}
$$

so that one can always choose the solution for which

$$
0 \leq \varphi \leq \pi,
$$

which explains the range of $\varphi$ given in Eq. (10).

Let us mention here that by performing a unitary transformation on the third qubit,

$$
\left|0^{\prime}\right\rangle=\frac{1}{\sqrt{\mu_{1}+\mu_{2}}}\left(\lambda_{1} e^{i \varphi}|0\rangle+\lambda_{2}|1\rangle\right),
$$


the decomposition for the second set of (2) is obtained. In the remainder we will use the first decomposition (10), which is physically and mathematically more convenient.

A generalization of the Schmidt decomposition is thus given by (10); any state can be written in this minimal form, generically in a unique way. The explicit algorithm for constructing this canonical form follows from the set of Eqs. (5)-(8). However, particular states can be obtained for different values of the five entanglement parameters. It is thus useful to have five independent invariants for the classification of states which we will obtain from (10). We will take here the five minimal polynomial invariants of [6].

$$
\begin{aligned}
& \text { Defining } \Delta \equiv\left|\lambda_{1} \lambda_{4} e^{i \varphi}-\lambda_{2} \lambda_{3}\right|^{2} \text { we find } \\
& \frac{1}{2} \leq I_{1} \equiv \operatorname{Tr} \rho_{A}^{2}=1-2 \mu_{0}\left(1-\mu_{0}-\mu_{1}\right) \leq 1 \text {, } \\
& \frac{1}{2} \leq I_{2} \equiv \operatorname{Tr} \rho_{B}^{2}=1-2 \mu_{0}\left(1-\mu_{0}-\mu_{1}-\mu_{2}\right) \\
& -2 \Delta \leq 1 \text {, } \\
& \frac{1}{2} \leq I_{3} \equiv \operatorname{Tr} \rho_{C}^{2}=1-2 \mu_{0}\left(1-\mu_{0}-\mu_{1}-\mu_{3}\right) \\
& -2 \Delta \leq 1 \text {, } \\
& \frac{1}{4} \leq I_{4} \equiv \operatorname{Tr}\left(\rho_{A} \otimes \rho_{B} \rho_{A B}\right) \\
& =1+\mu_{0}\left(\mu_{2} \mu_{3}-\mu_{1} \mu_{4}-2 \mu_{2}-3 \mu_{3}-3 \mu_{4}\right) \\
& -\left(2-\mu_{0}\right) \Delta \leq 1 \text {, } \\
& 0 \leq I_{5} \equiv\left|\operatorname{Hdet}\left(t_{i j k}\right)\right|^{2}=\mu_{0}^{2} \mu_{4}^{2} \leq \frac{1}{16} \text {, }
\end{aligned}
$$

where

$$
\begin{array}{cl}
\rho_{A B} \equiv \operatorname{Tr}_{C}|\Psi\rangle\langle\Psi| & \rho_{C} \equiv \operatorname{Tr}_{A B}|\Psi\rangle\langle\Psi| \\
\rho_{A} \equiv \operatorname{Tr}_{B} \rho_{A B} & \rho_{B} \equiv \operatorname{Tr}_{A} \rho_{A B}
\end{array}
$$

and Cayley's hyperdeterminant, $\operatorname{Hdet}\left(t_{i j k}\right)$, can be found in [7] and corresponds to the three-tangle of $[6,8]$.

Although these five invariants are computationally simple and physically meaningful, as they give local information, it can be convenient to trade them, recalling $\sum_{i} \mu_{i}=1$, for algebraically simpler ones:

$$
\begin{gathered}
0 \leq J_{1} \equiv \Delta \leq \frac{1}{4}, \\
0 \leq J_{2} \equiv \mu_{0} \mu_{2} \leq \frac{1}{4}, \\
0 \leq J_{3} \equiv \mu_{0} \mu_{3} \leq \frac{1}{4}, \\
0 \leq J_{4} \equiv \mu_{0} \mu_{4} \leq \frac{1}{4}, \\
J_{5} \equiv \mu_{0}\left(\Delta+\mu_{2} \mu_{3}-\mu_{1} \mu_{4}\right) .
\end{gathered}
$$

The invariants $J_{4}$ and $J_{5}$ are symmetric under permutation of parties, while $J_{1}\left(J_{2}, J_{3}\right)$ is symmetric under exchange of parties $B$ and $C$ ( $A$ and $C, A$ and $B$ ).

We can now proceed with the complete classification of nongeneric three-qubit states with the help of Eqs. (10) and (23):
Type 1 (product states): $J_{i}=0$ for $i=1,2,3,4,5$.

Type $2 \mathrm{a}$ (biseparable states): $J_{i}=0$ except $J_{1}\left(J_{2}, J_{3}\right)$ when party $A(B, C)$ is not entangled with the other two parties. They carry only bipartite entanglement and depend on one parameter.

Type $2 \mathrm{~b}$ (generalized GHZ states): $J_{i}=0$ except $J_{4}$. They include the standard GHZ states [9] and depend on one parameter.

Type 3a (tri-Bell states): $\mu_{1}=\mu_{4}=0$. It implies $J_{4}=$ $0, J_{1} J_{2}+J_{1} J_{3}+J_{2} J_{3}=\sqrt{J_{1} J_{2} J_{3}}=\frac{J_{5}}{2}$. They depend on two parameters.

Type 3b (extended GHZ states): $\mu_{j}=\mu_{k}=0$, for $j, k \in\{1,2,3\}$ and $j \neq k$. It implies $J_{j}=J_{k}=J_{5}=0$. They depend on two parameters and correspond to the slice states of [10].

Type 4a: $\mu_{4}=0$. It follows $J_{4}=0$ and $\sqrt{J_{1} J_{2} J_{3}}=\frac{J_{5}}{2}$. They depend on three parameters.

Type 4b: $\mu_{2}=0\left(\mu_{3}=0\right)$. Then, $J_{2}=J_{5}=0\left(J_{3}=\right.$ $\left.J_{5}=0\right)$. They depend on three parameters.

Type 4c: $\mu_{1}=0$. Then, $J_{1}\left(J_{2}+J_{3}+J_{4}\right)+J_{2} J_{3}=$ $\sqrt{J_{1} J_{2} J_{3}}=\frac{J_{5}}{2}$ and they depend on three parameters.

Notice that the type number indicates how many of the five states of (10) characterize the states of that type. Because of the asymmetric character of the decomposition (10), some of the states included in type 5 can be written in terms of four states, had we singled out party $B$ or $C$ [11]. Notice also that, in some sense, the $J_{i}$ 's are indicators of entanglement: only when all of them vanish there is no entanglement at all, $J_{1}\left(J_{2}, J_{3}\right)$ indicate bipartite entanglement, and $J_{4}$ indicates GHZ entanglement.

Let us further exploit our previous results. An alternative generalization of the Schmidt decomposition could be writing the state as a superposition of two nonorthogonal product states which are not built from local bases,

$$
|\Psi\rangle=\alpha|a b c\rangle+\beta\left|a^{\prime} b^{\prime} c^{\prime}\right\rangle
$$

with $\alpha$ and $\beta$ real.

Beside the trivial cases of type- 1 and type- 2 a states, this decomposition is always possible except for a familly of states depending on three parameters [12]. Our decomposition allows one to reproduce this result and shows that (24) is not possible when $I_{5}=0$ (corresponding to type$3 \mathrm{a}$ and type-4a states). It can be proved that when $I_{5}=0$ the two solutions of (8) coincide. The same happens had we chosen to single out any of the other parties. Therefore, for any party $X$, there is only one state $|0\rangle_{X}$ such that ${ }_{X}\langle 0 \mid \Psi\rangle$ is a product state of the other two parties. Since (24) implies two such states, e.g., $\left|a_{\perp}\right\rangle_{A}$ and $\left|a_{\perp}^{\prime}\right\rangle_{A}$, it follows that type-3a and type-4a states cannot be written as a sum of two nonorthogonal product states. When the decomposition (24) is possible, our results give the constructive method to obtain it. From (10), the second coefficient can be split into two terms,

$$
|\Psi\rangle=\left(\lambda_{0}|000\rangle+\frac{\lambda_{1} \lambda_{4} e^{i \varphi}-\lambda_{2} \lambda_{3}}{\lambda_{4}}|100\rangle\right)+\left(\frac{\lambda_{2} \lambda_{3}}{\lambda_{4}}|100\rangle+\lambda_{2}|101\rangle+\lambda_{3}|110\rangle+\lambda_{4}|111\rangle\right) .
$$


It is easy to see that (25) corresponds to the sum of two nonorthogonal product states as (24) with coefficients

$$
\begin{gathered}
\alpha=\frac{1}{\lambda_{4}} \sqrt{J_{1}+J_{4}}, \\
\beta=\frac{1}{\lambda_{4}} \sqrt{\mu_{2} \mu_{3}+\mu_{4}\left(\mu_{4}+\mu_{2}+\mu_{3}\right)} .
\end{gathered}
$$

This decomposition is unique. The states that appear in (24) are orthogonal to the ones that allow each party to destroy the entanglement between the other two parties with some nonvanishing probability.

A final consequence of (10) is that, by using the bipartite Schmidt decomposition, any pure state can be written as a superposition of a product state and a biseparable state, i.e.,

$$
|\Psi\rangle=\cos \theta|000\rangle+\sin \theta|1\rangle\left(\cos \omega\left|0^{\prime} 0^{\prime \prime}\right\rangle+\sin \omega\left|1^{\prime} 1^{\prime \prime}\right\rangle\right),
$$

which is the minimal decomposition in terms of orthogonal product states. It exhibits explicitly two of the five entanglement parameters. The other three are hidden in the moduli of the scalar products $\left\langle 0 \mid 0^{\prime}\right\rangle$ and $\left\langle 0 \mid 0^{\prime \prime}\right\rangle$, and in one phase absorbed by one of the local bases. It is also a nonsuperfluous form, though not built from local bases.

In this work we have found the minimal decomposition of any pure three-qubit state in terms of orthogonal product states built from local bases. It generalizes the Schmidt decomposition and leads to a complete classification of pure three-qubit states, which fine grains the fully inseparable states class of the general entanglement classification of mixed three-qubit states [13]. Our decomposition shows that any party can, performing a clever local measurement, kill the entanglement between the other two parties with nonvanishing probability. A decomposition in terms of the minimal number of orthogonal product states has also been found.

Finally, we have explored whether a pure three-qubit state can be written as a sum of two nonorthogonal product states, which can be thought of as an alternative gener- alization of the Schmidt decomposition. We have verified that only a subfamily depending on three parameters cannot be expressed in this form [12], corresponding to states with $I_{5}=0$.

The authors thank Guifré Vidal and Sandu Popescu for useful discussions. J.I. L. and R. T. acknowledge financial support by CICYT Project No. AEN 98-0431, CIRIT Project No. 1998SGR-00026, and CEC Project No. IST1999-11053, A. Andrianov by RFBR 99-01-00736 and CIRIT, PIV-2000, L. C. by PB97-0893, and A. Acín and E. J. by a grant from MEC (AP98 and AP99). Financial support from the ESF is also acknowledged.

*Email address: ejane@ecm.ub.es

[1] E. Schmidt, Math. Ann. 63, 433 (1907); A. Ekert and P. L. Knight, Am. J. Phys. 63, 415 (1995).

[2] A. Peres, Quantum Theory: Concepts and Methods (Kluwer Academic Publishers, Dordrecht, 1995); A. Peres, Phys. Lett. A 202, 16 (1995).

[3] A. V. Thapliyal, Phys. Rev. A 59, 3336 (1999); A. K. Pati, quant-ph/9911073.

[4] N. Linden and S. Popescu, Fortschr. Phys. 46, 567 (1998).

[5] J. Schlienz and G. Mahler, Phys. Lett. A 224, 39-44 (1996).

[6] A. Sudbery, quant-ph/0001116.

[7] I. M. Gelfand, M. M. Kapranov, and A. V. Zelevinsky, Discriminants, Resultants and Multidimensional Determinants (Birkhäuser, Boston, 1994). Its explicit form is $\operatorname{Hdet}\left(t_{i j k}\right)=t_{000}^{2} t_{111}^{2}+t_{001}^{2} t_{110}^{2}+t_{010}^{2} t_{101}^{2}+t_{100}^{2} t_{011}^{2}-$ $2\left(t_{000} t_{111} t_{011} t_{100}+t_{000} t_{111} t_{101} t_{010}+t_{000} t_{111} t_{110} t_{001}+\right.$ $\left.t_{011} t_{100} t_{101} t_{010}+t_{011} t_{100} t_{110} t_{001}+t_{101} t_{010} t_{110} t_{001}\right)+$ $4\left(t_{000} t_{110} t_{101} t_{011}+t_{111} t_{001} t_{010} t_{100}\right)$.

[8] V. Coffman, J. Kundu, and W. K. Wootters, quant-ph/ 9907047.

[9] D. M. Greenberger, M. A. Horne, and A. Zeilinger, Phys. Today 46, No. 8, 24 (1993).

[10] H. A. Carteret and A. Sudbery, quant-ph/0001091.

[11] A. Acín, A. Andrianov, E. Jané, J. I. Latorre, and R. Tarrach (to be published).

[12] J. I. Cirac, W. Dür, and G. Vidal, quant-ph/0005115.

[13] W. Dür, J. I. Cirac, and R. Tarrach, Phys. Rev. Lett. 83, 3562 (1999). 